\title{
Ab initio molecular dynamics study of dissociation of water under an electric field
}

\author{
A. Marco Saitta ${ }^{1}$ †, Franz Saija ${ }^{2}$ * , Paolo V. Giaquinta ${ }^{3}$ † \\ ${ }^{1}$ IMPMC, CNRS-UMR 7590, Université P \& $M$ Curie, 75252 Paris, France \\ 2 CNR-IPCF, Viale Ferdinando Stagno d'Alcontres 37, 98158 Messina, Italy \\ ${ }^{3}$ Università degli Studi di Messina, Dipartimento di Fisica, Contrada Papardo, 98166 Messina, Italy
}

(Dated: October 19, 2018)

\begin{abstract}
The behavior of liquid water under an electric field is a crucial phenomenon in science and engineering. However, its detailed description at a microscopic level is difficult to achieve experimentally. Here we report on the first $a b$ initio molecular-dynamics study on water under an electric field. We observe that the hydrogen-bond length and the molecular orientation are significantly modified at low-to-moderate field intensities. Fields beyond a threshold of about $0.35 \mathrm{~V} / \AA$ are able to dissociate molecules and sustain an ionic current via a series of correlated proton jumps. Upon applying even more intense fields $(\sim 1.0 \mathrm{~V} / \AA)$, a $15-20 \%$ fraction of molecules are instantaneously dissociated and the resulting ionic flow yields a conductance of about $7.8 \Omega^{-1} \cdot \mathrm{cm}^{-1}$, in good agreement with experimental values. This result paves the way to quantum-accurate microscopic studies of the effect of electric fields on aqueous solutions and, thus, to massive applications of $a b$ initio molecular dynamics in neurobiology, electrochemistry and hydrogen economy.
\end{abstract}

Water is the most abundant compound on Earth and plays a fundamental role in the realm of natural and life sciences, at the interface between a variety of disciplines such as physics, chemistry and biology [4, 5, with a seemingly infinite range of technological applications. Despite the interest and the enormous amount of fundamental and applied research work, a complete understanding of its unique behavior is still lacking [6]. The peculiar properties of water are due to its molecular structure and dipole moment and, as a consequence, to its hydrogen-bonding properties [7. Among the physicalchemical phenomena of greater interest, particularly relevant is the phenomenon of autoprotolysis, i.e. the dissociation of a water molecule according to the following reaction [8]:

$$
2 \mathrm{H}_{2} \mathrm{O} \rightleftharpoons \mathrm{OH}^{-}+\mathrm{H}_{3} \mathrm{O}^{+}
$$

where two molecules form a pair of hydronium and hydroxide ions which then separate, thus determining the $\mathrm{pH}$ of the substance. This effect is of paramount importance because it is a key step in all processes in which water is involved as more than just a passive solvent. The kinetics of the autoionization process has been studied in some experiments [9, but it is an extremely rare reaction which takes place on the femtosecond time scale of molecular motions [10, thus making its microscopic investigation very difficult. On the other hand, a deeper understanding of this reaction is crucially important in the presence of an external electric field, with consequences in disparate domains, from neurobiology [11] to electrolytic batteries [12] and hydrogen-based technology [13. For example, the activity of neurons is governed by the exchange of ions through the cell membrane, which is driven by the potential difference between the interior of the cell and its outside environment. The first experiments of forward and backward conversion of chemical energy into electric energy date back to 1789; the first battery was invented by Volta in 1800 and then used to carry out, for the first time, the electrolysis of water. Since then, electrochemistry has become a stand-alone branch of science and technology, with a huge impact on everyday life and more to come on the nascent hydrogen economy.

The chemical processes governing the autoprotolysis of water in the absence of an external field are in principle within the reach of $a b$ initio molecular-dynamics simulations. However, because of the large timescales involved, a straightforward molecular-dynamics approach turns out to be rather ineffective. The problem was overcome using a combination of Car-Parrinello (CP) molecular dynamics 14 and transition-path sampling [15, which confirmed that water dissociation is an extremely unfavored event from the energetic point of view. In a milestone study Geissler et al. showed that the rare autoprotolysis of a water molecule is induced by occasional large fluctuations of the electric field generated by the surrounding solvent, i.e. by other water molecules [16]. To test their theory, these authors adopted a clever scheme in which the occurrence of such rare fluctuations was favored by ad hoc semi-random moves of protons along hydrogen bonds; they showed that this method is equivalent to applying an external electric field and proved that such a field can dramatically change the energetic balance of the dissociation reaction, favoring the occurrence of the latter. The nascent hydronium and hydroxide ions then separate along a chain of hydrogen bonds via Grotthuss transfer events [17. It also turns out that their recombination is made possible through a collective compression of the water wire bridging the ions [18. However, when the hydrogen-bond chain still connecting the two ions breaks, their rapid recombination is inhibited and from such a state ions can move further apart and sep- 
arate completely. Based on this result, recent classical molecular-dynamics simulation studies have shown that the important contributions to the electric field acting on $\mathrm{OH}$ bonds stem from water molecules less than $7 \AA$ away, corresponding to charges on water molecules of the first and second hydration shell [19], and that they are able to generate instantaneous fields up to $2.5-3.0 \mathrm{~V} / \AA$.

Despite the major progress that such works represent in this domain, it has not been possible to establish, so far, a quantitative relation between the degree of water dissociation and the intensity of the electric field. Several studies based on the classical molecular dynamics of water molecules under an external electric field are already present in the literature, describing, for example, the effect of an electric field on the phase diagram [20] or the phenomenon of electrofreezing [21, 22. However, such studies are typically based on rigid-molecule non-polarizable potential models, which are unable to describe the dissociation of water molecules. On the other hand, the effect of an electric field in $a b$ initio Density Functional Theory (DFT) codes is traditionally taken into account through a sawtooth potential that is quite satisfactory in low-dimensional systems but which cannot be used in bulk systems. We present here the first, to our knowledge, $a b$ initio study of the properties of bulk water in the presence of intense external electric fields. The study was carried out using Quantum Espresso [23], a well-known open-source package for the DFT calculation of electronic-structure properties, which uses a plane-wave basis set and pseudopotentials. Macroscopic polarization and finite electric fields are treated by this code with the modern theory of polarization (Berry phases) 24. Previous ab initio calculations of water in an external field were typically carried out on small clusters in the vacuum [25. All the technical details of the calculation are reported in the Supplementary Material file [26].

In order to test the reliability of the method, we first checked the effect of a uniform electric field by calculating the field-induced electronic polarization at fixed atomic positions for several water configurations as in Ref. 24; the estimate we obtained $\left(\epsilon_{\infty} \approx 1.74\right)$ is in good agreement with the experimental data as well as with previous theoretical results (we refer the reader to Ref. [26] for more detailed information). We also calculated the structural properties of the system at zero field, such as the radial distribution functions, and found that they reproduce with fair accuracy those reported in the literature [26]. As shown in Ref. [27], the evolution of the structure of water and of its H-bonding properties are better described by the instantaneous distribution of the distance between an oxygen atom and its third hydrogen neighbor, i.e. typically the closest $\mathrm{H}$ neighbor covalently bonded to a nearby oxygen atom. We report such a distribution, for values of the external electric field up to $1 \mathrm{~V} / \AA$, in the top panel of Fig. 1. to-
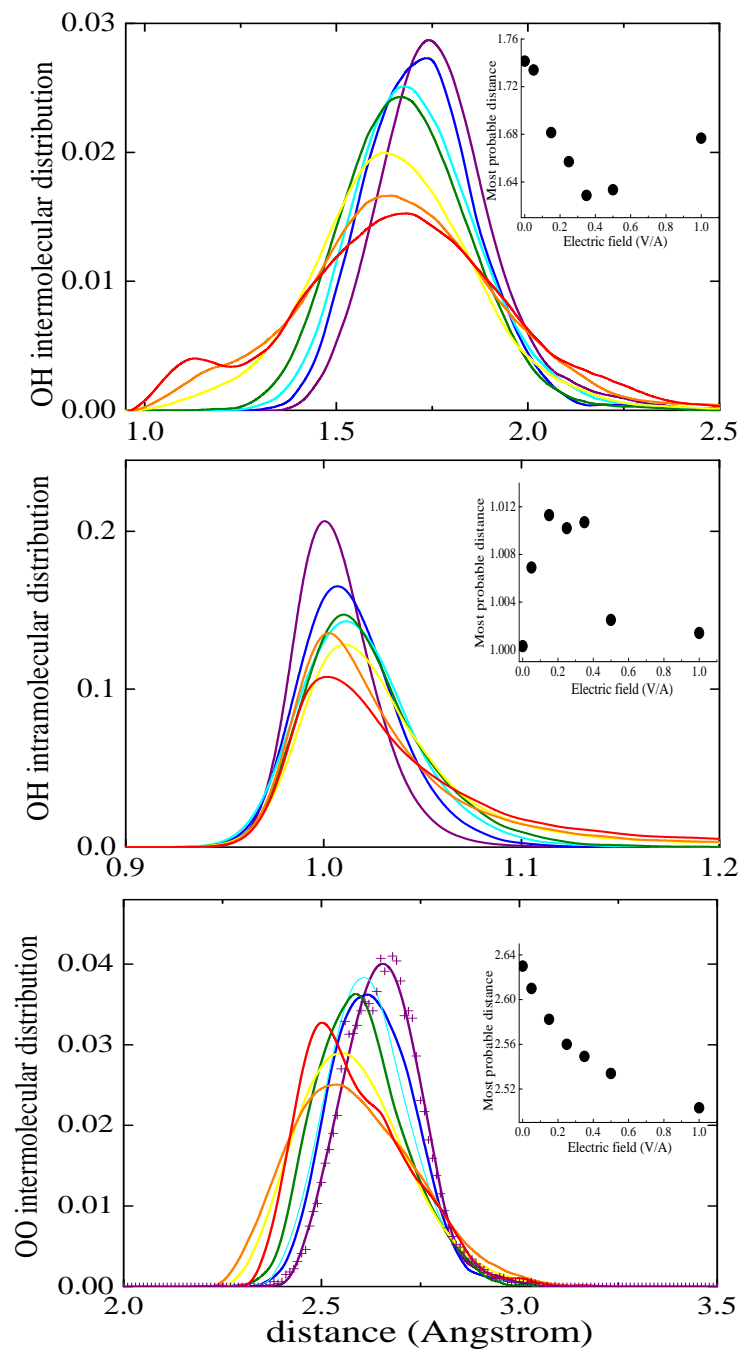

FIG. 1: Distributions of istantaneous $\mathrm{O}-\mathrm{O}$ and $\mathrm{O}-\mathrm{H}$ distances for electric fields in the range between zero (purple curves) and $1 \mathrm{~V} / \AA$ (red curves). The insets show the evolution of most probable distances with the field intensity. Top panel: intermolecular distance between an oxygen atom and its third hydrogen neighbor; central panel: intramolecular distance between an oxygen atom and its second hydrogen neighbor; bottom panel: oxygen-oxygen closest-neighbor distance. The experimental data were smoothed with the Savitzky-Golay filter method, as typically shown for the zero-field curve in the bottom panel.

gether with the intramolecular $\mathrm{O}-\mathrm{H}$ distance distribution of the second hydrogen neighbor (central panel), and the $\mathrm{O}-\mathrm{O}$ closest-neighbor distance distribution (bottom panel). The effects produced by increasing electric fields on the first distribution (top panel) are a systematic broadening and a significant shortening of the 

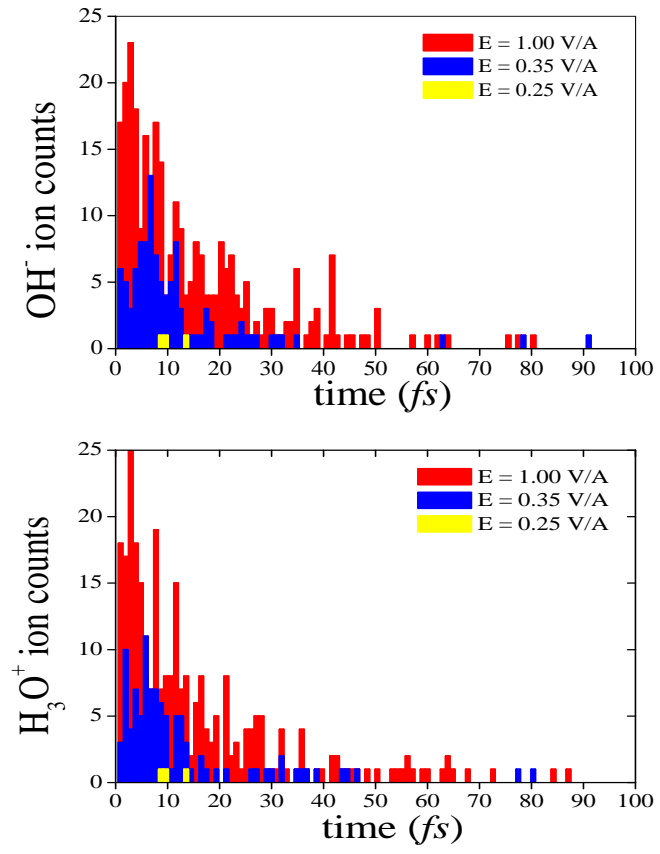

FIG. 2: Counts of $\mathrm{OH}^{-}$and $\mathrm{H}_{3} \mathrm{O}^{+}$ionic species with lifetimes as abscissæ, for field intensities $0.25 \mathrm{~V} / \AA$ (yellow), $0.35 \mathrm{~V} / \AA$ (blue), and $1 \mathrm{~V} / \AA$ (red).

H-bond for fields up to about $0.35 \mathrm{~V} / \AA$; for larger intensities, the maximum of the distribution weakly shifts again to larger and larger values while a secondary peak emerges at about $1.1 \AA$, which can be associated to a covalently bonded hydrogen in a $\mathrm{H}_{3} \mathrm{O}^{+}$ionic species. The effect of the field is also manifest on the intramolecular covalent bond between an oxygen atom and its second hydrogen neighbor, as shown in the central panel of Fig. 1: the associated distribution becomes more and more delocalized while the most probable distance exhibits a nonmonotonic trend, opposite to that observed above for the hydrogen bond. Note also, in the bottom panel of Fig. 1, the monotonic shortening of the $\mathrm{O}-\mathrm{O}$ distance up to an amount of about $0.14 \AA$, a shift comparable to that observed in water under a pressure of about $20 \mathrm{GPa} 28$. In classical simulations intermolecular distances are unaffected while an increased number of H-bonds has been observed with longer lifetimes [21. We interpret this partial discrepancy as a clear indication of a larger, field-induced $\mathrm{O}-\mathrm{H}$ intramolecular polarization, which strengthens the intermolecular $\mathrm{O}-\mathrm{H}$ interaction and which cannot be accounted for in the classical models most commonly used to describe water. We also observed, indeed rather unsurprisingly as this effect has been already predicted by classical simulations 21, that water molecules tend to align their dipoles along a direction parallel to the field [26].

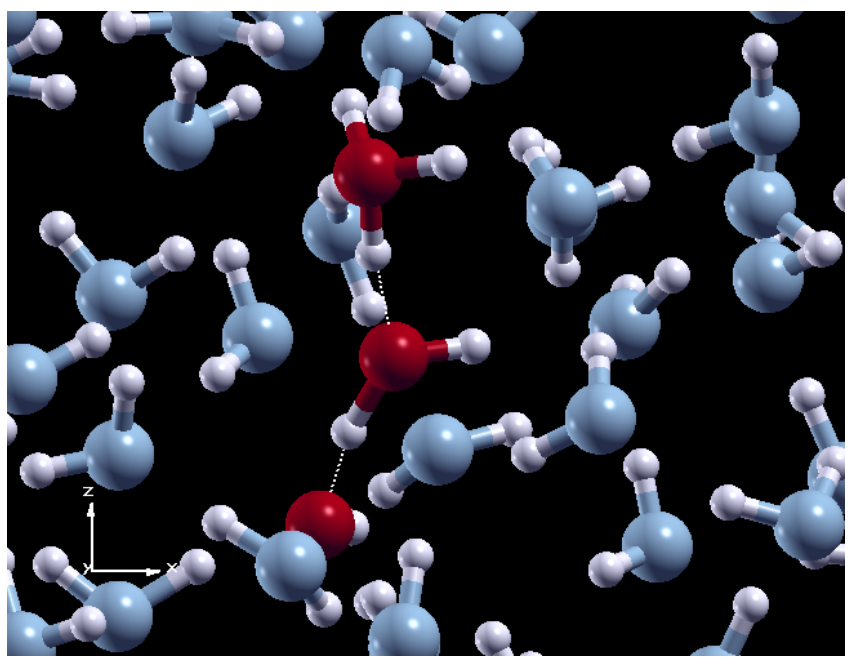

FIG. 3: Snapshot of a typical dissociation-diffusion mechanism; the molecules involved in the process are rendered with red coloring, while the surrounding molecules have graycolored oxygen atoms. In both cases, hydrogens are depicted in white. In particular, a $\mathrm{H}_{3} \mathrm{O}^{+}-\mathrm{H}_{2} \mathrm{O}-\mathrm{OH}^{-}$entity is clearly visible and its relevant hydrogen-bonds are indicated with a dashed white line. The electric field is oriented along the positive $z$ direction.

According to the present calculations, water molecules start to dissociate for field intensities around $0.25 \mathrm{~V} / \AA$. Protons participating in H-bonds, mostly aligned with the external field, start to jump occasionally back and forth along the bond, thus creating instantaneous $\mathrm{H}_{3} \mathrm{O}^{+}-\mathrm{OH}^{-}$pairs. Such events are still rare and produce extremely short-lived $(\lesssim 10 f s)$ ionic pairs, the field being not strong enough to permanently sustain instantaneous ionic dipoles for times long enough to start a real proton diffusion (see Fig. 22). Only for fields of about 0.35 $\mathrm{V} / \AA$ we started to observe much more frequent molecular dissociations, suggesting that the balance of the reaction described in Eq. 1 more and more favors the products in the right term at the expense of the reactants in the left term, i.e. the neutral state. We note that the above field threshold is in very good agreement with experiments. In a work of a few years ago [30, water dissociation was observed to occur under external fields of 0.32 and 0.44 $\mathrm{V} / \AA$, the threshold depending on the temperature. A very recent experimental study provided a thorough review of the process of water ionization in high electric fields induced by Pt electrodes 31. In particular, an interesting analysis of the interplay between the local field, as estimated by Geissler et al. [16, and the external field concludes that the latter should enhance water ionization for intensities of $0.3-0.6 \mathrm{~V} / \AA$, in excellent agreement with our results.

Once the external field is strong enough to induce a significant dissociation, ionic lifetimes become dramatically longer, ranging from a few femtoseconds to more 
than $0.1 \mathrm{ps}$ for both $\mathrm{H}_{3} \mathrm{O}^{+}$and $\mathrm{OH}^{-}$, as reported in Fig. 2. Indeed, at this point the dissociation reactions are typically followed by a series of proton jumps from the newly formed hydronium to the adjacent H-bonded neutral molecule along the field direction, forming a new hydronium from which a (different) proton can jump, and so on and so forth. In other words, ionic diffusion (and thus conduction) is activated by the dissociation reaction described in Eq. (1), but the presence of the field breaks the symmetry of the physical space, providing a spatial direction for the relevant proton hopping events, and thus favoring (or disfavoring) their respective reaction equilibria according to the relative physical position of the reactants, with respect to each other and to the field. This effect can be visually pictured as follows:

$$
\mathrm{H}_{2} \mathrm{O}+\mathrm{H}_{2} \mathrm{O} \underset{E}{\longrightarrow} \mathrm{OH}^{-}+\mathrm{H}_{3} \mathrm{O}^{+}
$$

where the right-directed oriented field induces a proton jump from the left water molecule to the right one. By analogy, beyond the dissociation threshold the recombination processes more likely occurs as:

$$
\mathrm{H}_{3} \mathrm{O}^{+}+\mathrm{OH}^{-} \underset{E}{\longrightarrow} \mathrm{H}_{2} \mathrm{O}+\mathrm{H}_{2} \mathrm{O}
$$

i.e. the extra proton comes from an hydronium in the opposite direction of the hydroxide with respect to the field. Besides these two steps, ionic conduction is sustained by other processes which can be decomposed in terms of the following elementary proton jumps:

$$
\mathrm{H}_{3} \mathrm{O}^{+}+\mathrm{H}_{2} \mathrm{O} \underset{E}{\longrightarrow} \mathrm{H}_{2} \mathrm{O}+\mathrm{H}_{3} \mathrm{O}^{+}
$$

and

$$
\mathrm{H}_{2} \mathrm{O}+\mathrm{OH}^{-} \underset{E}{\longrightarrow} \mathrm{OH}^{-}+\mathrm{H}_{2} \mathrm{O}
$$

where, again, proton hopping along the field direction is privileged. We note that proton jumps are significantly correlated; in particular, protons originally forming donor H-bonds and belonging to adjacent molecules jump almost simultaneously as their respective acceptor molecule, in a Zundel-to-Zundel mechanism very similar to the one described in ab-initio simulations [16] as well as in experiments 29]. The mechanism of correlated proton jump diffusion is illustrated by a snapshot of our simulation cell in Fig. 3. Dissociated molecules recombine with protons diffusing from neighboring molecules, thus contributing to the global liquid conductivity. We observed that even for fields of $1.0 \mathrm{~V} / \AA$ adjacent $\mathrm{H}_{3} \mathrm{O}^{+}-\mathrm{OH}^{-}$ pairs are not viable, unless they are separated by an $\mathrm{H}_{2} \mathrm{O}$ molecule, and that the Zundel-to-Zundel diffusion mechanism is dominant. Such correlated processes can involve even more than two protons at the same time, but still the decomposition of the diffusion mechanism in the individual elementary steps of Eqs. (2, 5) is possible, and allows a more quantitative description of the whole phenomenon and of the corresponding rates. To this end, we reported in Table I the number of such elementary events per molecule and per picosecond. We note that at the dissociation threshold $(0.35 \mathrm{~V} / \AA)$ a single molecule undergoes, on average, about 3 proton-hopping events in a timespan of 1 picosecond, about 6 at $0.5 \mathrm{~V} / \AA$, up to about 10 at the highest field considered in this work, and that the fraction of instantaneously ionized molecules $\Delta N$ increases accordingly. In particular, at the most in-

\begin{tabular}{crrrrc}
\hline \hline Field $(\mathrm{V} / \AA)$ & Eq. $(2)$ & Eq. $(3)$ & Eq. $(4)$ & Eq. $(5)$ & $\Delta N$ \\
\hline 0.25 & 0.148 & 0.135 & 0.067 & 0.108 & 0.0046 \\
0.35 & 0.952 & 0.872 & 0.743 & 0.823 & 0.0408 \\
0.50 & 1.211 & 1.049 & 1.808 & 1.856 & 0.0828 \\
1.00 & 2.341 & 2.082 & 2.502 & 2.728 & 0.1556 \\
\hline \hline
\end{tabular}

TABLE I: Calculated event rates and ion fractions for different field intensities, per molecule and per picosecond of dynamical evolution: dissociation (Eq.(2)); recombination (Eq. (3p); hydronium-to-water hopping (Eq.44); water-tohydroxide hopping (Eq. (5)). The average fraction of ionized molecules is reported in the last column.

tense field a fraction $\Delta N \approx 0.15-0.20$ of the total number of molecules are instantaneously ionic, as appears from the rightmost column of Table I] giving rise to a ohmic conductivity of about $7.8 \Omega^{-1} \cdot \mathrm{cm}^{-1}$; this latter finding is in fairly good agreement with the available experimental values 32,34 of water dissociation under pressures larger than $20 \mathrm{GPa}$, as analogously observed in $a b$ initio $\mathrm{CP}$ calculations carried out in similar extreme conditions [28, 35]. In particular, the authors of Refs. 33] and 34 observed in shock-wave experiments a conductivity of about $30 \Omega^{-1} \cdot \mathrm{cm}^{-1}$, which they attributed to a massive presence of ionic species; they also estimated that fully ionized water (i.e. $100 \%$ of dissociated molecules) should have, at those regimes, a conductivity of about $36 \Omega^{-1} \cdot \mathrm{cm}^{-1}$. Our result thus seems to indicate that strong electric fields, yet similar to those instantaneously generated in neutral water by the surrounding molecules [19, are able to generate ionic species and to induce an ionic current whose intensity is comparable to that observed in different experimental conditions and setups. The calculated ionic current-voltage characteristic of water is finally presented in Fig. 4 .

We conclude that the ab initio Car-Parrinello approach is able to catch the essential features of molecular dissociation and diffusion mechanisms of bulk water under the action of an intense electric field. The results illustrated in this Letter show that the method employed not only efficaciously describes the dissociation of the molecule but further accounts for the presence of the forming ionic species whose effect, even if short lived, is very important in the dynamics of ions in solution. It appears that $a b$ initio simulations of aqueous/molecular systems under 


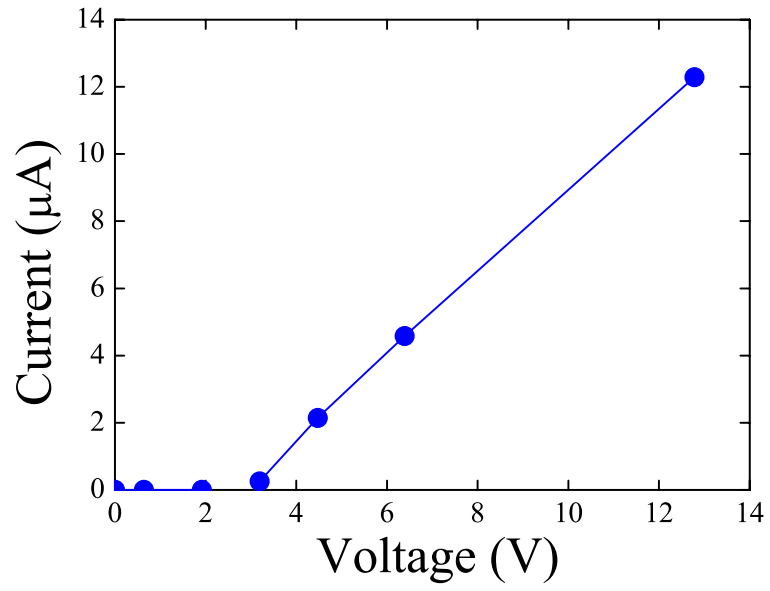

FIG. 4: Ionic current-voltage characteristic for a cubic cell of $12.42 \AA$ side. The dots represent the calculated values, while the solid line is a guide for the eye.

an electric field are now feasible with a numerical accuracy which may already allow for a comparison with the available experimental data. A stimulating scenario of new investigations opens up with a range of applications of potentially enormous reach, from planetary science, to biology, electrochemistry and the fast-growing field of hydrogen-based economy.

\section{Acknowledgements}

We acknowledge the IDRIS Supercomputing Facility for CPU time (CP9-101387).

[†] Corresponding author. E-mail: marco.saitta@impmc.upmc.fr

[*] E-mail: saija@me.cnr.it

[†] E-mail: paolo.giaquinta@unime.it

[4] A. Ben-Naim, Molecular Theory of Water and Aqueous Solutions - Part 1: Understanding Water (World Scientific: Singapore, 2009).

[5] P. Ball, in $\mathrm{H}_{2} \mathrm{O}:$ A biography of water, (Phoenix, London, ed. 5, 2004).

[6] P. Ball, Chem. Rev. 108, 74 (2008).

[7] M. Gerstein, M. Levitt, Sci. Am. 279, 100 (May 1998).
[8] IUPAC. Compendium of Chemical Terminology, 2nd ed. (the "Gold Book"). Compiled by A. D. McNaught and A. Wilkinson. Blackwell Scientific Publications, Oxford (1997).

[9] W. C. Natzle, C. B. Moore, J. Phys. Chem. B 89, 2605 (1985).

[10] M. Eigen, L. De Maeyer, Z. Electrochem. 59, 986 (1955).

[11] K. Kaila, B. R. Ransom, in $p H$ and brain function, K. Kaila, B. R. Ransom, eds. (Wiley, New York, 1998).

[12] J. O'M. Bockris, A. K. N. Reddy, in Modern Electrochemistry, (Kluwer Academic, New York, 2000).

[13] E. I. Zoulias, N. Lymberopoulos, in Hydrogen-based Autonomous Power Systems, (Springer, London, 2008).

[14] R. Car, M. Parrinello, Phys. Rev. Lett. 55, 2471 (1985).

[15] C. Dellago, P. G. Bolhuis, P. L. Geissler, Adv. Chem. Phys. 123, 1 (2002).

[16] P. L. Geissler, C. Dellago, D. Chandler, J. Hutter, M. Parrinello, Science 291, 2121 (2001).

[17] C. J. T. de Grotthuss, Ann. Chim. 58, 54 (1806).

[18] A. Hassanali, M. K. Prakash, H. Heshet, and M. Parrinello, Procl. Nat. Acad. Sci. USA 108, 20410 (2012).

[19] B. Reischl, J. Kofinger, C. Dellago, Mol. Phys. 107, 495 (2009).

[20] J. L. Aragones, L. G. MacDowell, J. I. Siepmann, and C. Vega, Phys. Rev. Lett. 107, 155702 (2011).

[21] S. Wei, C. Zhong, H. Su-Yi, Mol. Simul. 31, 555 (2005).

[22] I.M. Svishchev and P.G. Kusalik, J. Am. Chem. Soc. 118, 649 (1996).

[23] P. Giannozzi et al., J. Phys. Condens. Matter 21, 395502 (2009).

[24] P. Umari, A. Pasquarello, Phys. Rev. Lett. 89, 157602 (2002).

[25] Y. C. Choi, C. Pak, K. S. Kim, J. Chem. Phys. 124, 094308 (2006).

[26] See EPAPS Document No. For more information on EPAPS, see http://www.aip.org/pubservs/epaps.html

[27] A. M. Saitta, F. Datchi, Phys. Rev. E (RC) 67, 020201(R) (2003).

[28] E. Schwegler, G. Galli, F. Gygi, R. Q. Hood, Phys. Rev. Lett. 87, 265501 (2001).

[29] S. T. Roberts, P. B. Petersen, K. Ramasesha, A. Tokmakoff, I. S. Ufimtsev, and T. J. Martinez, PNAS 106, 15154 (2009).

[30] C. J. Rothfuss, V. K. Medvedev, E. M. Stuve, J. Electroanal. Chem. 554-555, 133 (2003).

[31] E. M. Stuve, Chem. Phys. Lett. 519-520, 1 (2012).

[32] S. D. Hamann and M. Linton, Trans. Faraday Soc. 62, 2234 (1966).

[33] R. Chau, A. C. Mitchell, R. W. Minich, W. J. Nellis, J. Chem. Phys, 114, 1361 (2001).

[34] R. Chau, S. Hamel, W. J. Nellis, Nat. Commun. 2, 203 (2011).

[35] C. Cavazzoni, G. L. Chiarotti, S. Scandolo, E. Tosatti, M. Bernasconi, and M. Parrinello Science 283, 44 (1999). 